\title{
Design of Exponential State Estimators for Neural Networks with Mixed Time Delays
}

\author{
Yurong Liu, Zidong Wang* and Xiaohui Liu
}

\begin{abstract}
In this paper, the state estimation problem is dealt with for a class of recurrent neural networks (RNNs) with mixed discrete and distributed delays. The activation functions are assumed to be neither monotonic, nor differentiable, nor bounded. We aim at designing a state estimator to estimate the neuron states, through available output measurements, such that the dynamics of the estimation error is globally exponentially stable in the presence of mixed time delays. By using the Laypunov-Krasovskii functional, a linear matrix inequality (LMI) approach is developed to establish sufficient conditions to guarantee the existence of the state estimators. We show that both the existence conditions and the explicit expression of the desired estimator can be characterized in terms of the solution to an LMI. A simulation example is exploited to show the usefulness of the derived LMI-based stability conditions.
\end{abstract}

\section{Keywords}

State estimator; Recurrent neural networks; Discrete and distributed delays; Lyapunov-Krasovskii functional; Linear matrix inequality.

\section{INTRODUCTION}

The last few decades have witnessed a large amount of successful applications of neural networks in various areas including image processing, pattern recognition, associative memory, and optimization problems. In particular, high-order and large-scale neural networks have shown their great capacities in learning and data handling. For relatively high-order and large-scale neural networks, however, it is often the case that only partial information about the neuron states is available in the network outputs. Therefore, in order to make use of the neural networks in practice, it becomes necessary to estimate the neuron states through available measurements. The state estimation problem for neural networks has recently drawn particular research interests, see $[5,8,14,18]$. For example, in [14], an adaptive state estimator has been described by using techniques of optimization theory, the calculus of variations and gradient descent dynamics. In [18], the neuron state estimation problem has been addressed for recurrent neural networks with time-varying delays, and an effective LMI approach has been developed to verify the stability of the estimation error dynamics.

On the other hand, many biological and artificial neural networks contain inherent time delays in signal transmission, which may cause oscillation and instability (see e.g. $[1,12,18]$ ). In recent years, a great number of papers have been published on various neural networks with time delays, and the existence of equilibrium point, global asymptotic stability, global exponential stability, and the existence of periodic solutions have been intensively investigated, see [3,4,10,15-17,19-24] for some recent results.

For the dynamical behavior analysis of delayed neural networks, different types of time delays, such as constant delays, time-varying delays, and distributed delays, have been taken into account by using a variety

This work was supported in part by the Engineering and Physical Sciences Research Council (EPSRC) of the U.K. under Grant GR/S27658/01, the Nuffield Foundation of the U.K. under Grant NAL/00630/G, the Alexander von Humboldt Foundation of Germany, the Natural Science Foundation of Jiangsu Education Committee of China under Grant 05KJB110154, and the National Natural Science Foundation of China under Grant 10471119.

Y. Liu is with the Department of Mathematics, Yangzhou University, Yangzhou 225002, P. R. China.

Z. Wang and X. Liu are with the Department of Information Systems and Computing, Brunel University, Uxbridge, Middlesex, UB8 3PH, United Kingdom.

*All correspondences concerning this paper should be addressed to Z. Wang. (Email: Zidong.Wang@brunel.ac.uk) 
of techniques that include linear matrix inequality (LMI) approach, Lyapunov functional method, $M$-matrix theory, topological degree theory, and techniques of inequality analysis. For example, most recently, in [16], sufficient conditions, which ensure the existence and uniqueness of the equilibrium point and global exponential stability of bi-directional associative memory (BAM) neural networks with distributed delays and reactiondiffusion terms, are obtained by using the theory of topological degree, properties of M-matrix and Lyapunov functional. In [2], several novel sufficient criteria are given, in terms of matrix inequalities, for checking the global robust stability of equilibria for interval neural networks with time delays based on Lyapunov method and linear matrix inequality (LMI) technique. In [19-21], the global asymptotic stability analysis problem has been dealt with for a class of neural networks with discrete and distributed time-delays by using an effective LMI approach.

Up to now, comparing to the huge volume of literature on analyzing dynamical behavior analysis of delayed neural networks, the state estimation problem for generalized RNNs with both discrete and distributed timedelays have received relatively little research attention, despite its important application potential. This situation motivates us, in this paper, to investigate the state estimation problem for a class of neural networks with discrete and distributed time-delays. The purpose of the problem is to estimate the neuron states via available output measurements such that the estimation error converges to zero exponentially. A numerically efficient LMI approach is developed to solve the addressed problem, and the explicit expression of the set of desired estimators is characterized. A simulation example is used to demonstrate the usefulness of the LMI method.

\section{Problem Formulation}

Notations: The notations are quite standard. Throughout this paper, $\mathbb{R}^{n}$ and $\mathbb{R}^{n \times m}$ denote, respectively, the $n$-dimensional Euclidean space and the set of all $n \times m$ real matrices. The superscript " $T$ " denotes matrix transposition and the notation $X \geq Y$ (respectively, $X>Y$ ) where $X$ and $Y$ are symmetric matrices, means that $X-Y$ is positive semidefinite (respectively, positive definite). $I_{n}$ is the $n \times n$ identity matrix. $|\cdot|$ is the Euclidean norm in $\mathbb{R}^{n}$. If $A$ is a matrix, denote by $\|A\|$ its operator norm, i.e., $\|A\|=\sup \{|A x|:|x|=1\}=$ $\sqrt{\lambda_{\max }\left(A^{T} A\right)}$ where $\lambda_{\max }(\cdot)$ (respectively, $\lambda_{\min }(\cdot)$ ) means the largest (respectively, smallest) eigenvalue of $A$. Sometimes, the arguments of a function or a matrix will be omitted in the analysis when no confusion can arise. Furthermore the standard symbol $C\left([a, b] ; \mathbb{R}^{n}\right)$ denotes the set of continuous vector-valued functions defined on the interval $[a, b]$.

Consider the following recurrent neural network with mixed time delays:

$$
\frac{d x_{i}(t)}{d t}=-d_{i} x_{i}(t)+\sum_{j=1}^{n} a_{i j} f_{j}\left(x_{j}(t)\right)+\sum_{j=1}^{n} b_{i j} g_{j}\left(x_{j}\left(t-\tau_{1}\right)\right)+\int_{t-\tau_{2}}^{t} \sum_{j=1}^{n} w_{i j} h_{j}\left(u_{j}(s)\right) d s+I_{i}(t), \quad i=1, \ldots, n
$$

where $n$ is the number of the neurons in the neural network, $x_{i}(t)$ denotes the state of the $i$ th neural neuron at time $t, f_{j}(\cdot), g_{j}(\cdot)$ and $h_{j}(\cdot)$ are the activation functions of the $j$ th neuron. The constants $a_{i j}, b_{i j}$ and $w_{i j}$ denote, respectively, the connection weights, the discretely delayed connection weights, and the distributively delayed connection weights, of the $j$ th neuron on the $i$ neuron. $I_{i}(t)$ is the external time-varying bias on the $i$ th neuron, $d_{i}$ denotes the rate with which the $i$ th neuron will reset its potential to the resting state in isolation when disconnected from the network and external inputs. $\tau_{1}$ is the constant discrete time delay, while $\tau_{2}$ describes the distributed time delay.

The neural network (1) can be rewritten as the following matrix-vector form:

$$
\frac{d x(t)}{d t}=-D x(t)+A F(x(t))+B G\left(x\left(t-\tau_{1}\right)\right)+W \int_{t-\tau_{2}}^{t} H(x(s)) d s+I(t),
$$


where $x(t)=\left[x_{1}(t), x_{2}(t), \cdots, u_{n}(t)\right]^{T}, D=\operatorname{diag}\left(d_{1}, \ldots, d_{n}\right), A=\left(a_{i j}\right)_{n \times n}, B=\left(b_{i j}\right)_{n \times n}, W=\left(w_{i j}\right)_{n \times n}$, $I(t)=\left(I_{1}(t), \ldots, I_{n}(t)\right)^{T}$, and $F(x(t))=\left(f_{1}\left(x_{1}(t)\right), \ldots, f_{n}\left(x_{n}(t)\right)\right)^{T}, G\left(x\left(t-\tau_{1}\right)\right)=\left(g_{1}\left(x_{1}\left(t-\tau_{1}\right)\right), \ldots, g_{n}\left(u_{n}(t-\right.\right.$ $\left.\left.\left.\tau_{1}\right)\right)\right)^{T}, H(x(s))=\left(h_{1}\left(x_{1}(s)\right), \ldots, h_{n}\left(x_{n}(s)\right)\right)^{T}$.

Traditionally, the activation functions are assumed to be continuous, differentiable, monotonically increasing and bounded, such as the sigmoid-type of function. However, as discussed in [3, 4], in many electronic circuits, the input-output functions of amplifiers may be neither monotonically increasing nor continuously differentiable, hence nonmonotonic functions can be more appropriate to describe the neuron activation in designing and implementing an artificial neural network. In this paper, we make following assumption for the neuron activation functions, where the activation functions no longer need to be differentiable, monotonically increasing and bounded.

Assumption 1: For $i \in\{1,2, \ldots, n\}$, the neuron activation functions in (2) satisfy

$$
\begin{aligned}
& l_{i}^{-} \leq \frac{f_{i}\left(s_{1}\right)-f_{i}\left(s_{2}\right)}{s_{1}-s_{2}} \leq l_{i}^{+}, \\
& \sigma_{i}^{-} \leq \frac{g_{i}\left(s_{1}\right)-g_{i}\left(s_{2}\right)}{s_{1}-s_{2}} \leq \sigma_{i}^{+}, \\
& v_{i}^{-} \leq \frac{h_{i}\left(s_{1}\right)-h_{i}\left(s_{2}\right)}{s_{1}-s_{2}} \leq v_{i}^{+},
\end{aligned}
$$

where $l_{i}^{-}, l_{i}^{+}, \sigma_{i}^{-}, \sigma_{i}^{+}, v_{i}^{-}, v_{i}^{+}$are some constants.

Remark 1: The constants $l_{i}^{-}, l_{i}^{+}, \sigma_{i}^{-}, \sigma_{i}^{+}, v_{i}^{-}, v_{i}^{+}$in Assumption 1 are allowed to be positive, negative or zero. Hence, the resulting activation functions could be non-monotonic, and more general than the usual sigmoid functions. It is also noted that, for the state estimation task addressed in this paper, the neuron activation functions in (2) are not assumed to be bounded as usual.

It is worth noticing that for either biological or artificial neural networks, it is usually the case that the state of the neural network is not completely accessible and all the information one can have is just the output of the neural network. Subsequently, estimating the neuron state from the given output is necessary to realize some specific design objectives in many practical applications, and there is a need to construct an estimator to approximate the state of the neural network (2) in an asymptotical or exponential way.

Suppose that the output from the neural network (2) is of the form:

$$
y(t)=C x(t)+Q(t, x(t)) .
$$

Here, $y(t)=\left(y_{1}(t), \ldots, y_{m}(t)\right)^{T} \in \mathbb{R}^{m}$ is the measurement output of the neural network, $C \in \mathbb{R}^{m \times n}$ is a known constant matrix, and $Q(t, x(t))=\left(q_{1}(t, x(t)), \ldots, q_{m}(t, x(t))\right)^{T} \in \mathbb{R}^{m}$ is the nonlinear disturbance dependant on the neuron state that satisfies the following Lipschitz condition:

$$
|Q(t, x)-Q(t, y)| \leq|R(x-y)|
$$

where $R \in \mathbb{R}^{n \times n}$ is a known constant matrix.

In order to estimate the neuron state of (2), we construct the following full-order state estimator:

$$
\frac{d \hat{x}(t)}{d t}=-D \hat{x}(t)+A F(\hat{x}(t))+B G\left(\hat{x}\left(t-\tau_{1}\right)\right)+W \int_{t-\tau_{2}}^{t} H(\hat{x}(s)) d s+I(t)+K[y(t)-C \hat{x}(t)-Q(t, \hat{x}(t))],
$$

where $\hat{x}(t)$ is the state estimate, and $K \in \mathbb{R}^{n \times m}$ is the estimator gain matrix to be designed.

Our aim is to choose a suitable $K$ so that $\hat{x}(t)$ approaches $x(t)$ asymptotically or exponentially. For this purpose, we let $\mathcal{E}(t)=\left(\epsilon_{1}(t), \epsilon_{2}(t), \ldots, \epsilon_{n}(t)\right)^{T}:=\hat{x}(t)-x(t)$ be the state estimation error. Then in terms of $(2),(6)$ and (8), the state error $e(t)$ satisfies the following equation

$$
\frac{d \mathcal{E}(t)}{d t}=(-D-K C) \mathcal{E}(t)+A \hat{F}(\mathcal{E}(t))+B \hat{G}\left(\mathcal{E}\left(t-\tau_{1}\right)\right)+W \int_{t-\tau_{2}}^{t} \hat{H}(\mathcal{E}(s)) d s-K \hat{Q}(t, \mathcal{E}(t))
$$


where, for notation simplicity, we denote

$$
\begin{aligned}
& \hat{F}(\mathcal{E}(t))=\left[\hat{f}_{1}\left(\epsilon_{1}(t)\right), \hat{f}_{2}\left(\epsilon_{2}(t)\right) \ldots, \hat{f}_{n}\left(\epsilon_{n}(t)\right)\right]^{T}:=F(\hat{x}(t))-F(x(t)), \\
& \hat{G}(\mathcal{E}(t))=\left[\hat{g}_{1}\left(\epsilon_{1}(t)\right), \hat{g}_{2}\left(\epsilon_{2}(t)\right), \ldots, \hat{g}_{n}\left(\epsilon_{n}(t)\right)\right]^{T}:=G(\hat{x}(t))-G(x(t)), \\
& \hat{H}(\mathcal{E}(t))=\left[\hat{h}_{1}\left(\epsilon_{1}(t)\right), \hat{h}_{2}\left(\epsilon_{2}(t)\right), \ldots, \hat{h}_{n}\left(\epsilon_{n}(t)\right)\right]^{T}:=H(\hat{x}(t))-H(x(t)), \\
& \hat{Q}(t, \mathcal{E}(t)):=Q(t, \hat{x}(t))-Q(t, x(t)) .
\end{aligned}
$$

Notice that $\hat{F}(\mathcal{E}(t)), \hat{G}(\mathcal{E}(t)), \hat{H}(\mathcal{E}(t))$ and $\hat{Q}(t, \mathcal{E}(t))$ are all dependant on $x(t)$ or $\hat{x}(t)$, as well as $\mathcal{E}(t)$. In order to avoid cumbersome notations, we just use the symbols $\hat{F}(\mathcal{E}(t)), \hat{G}(\mathcal{E}(t), \hat{H}(\mathcal{E}(t))$ and $\hat{Q}(t, \mathcal{E}(t))$ to represent $\hat{F}(\mathcal{E}(t), x(t)), \hat{G}(\mathcal{E}(t), x(t)), \hat{H}(\mathcal{E}(t), x(t))$ and $\hat{Q}(t, \mathcal{E}(t), x(t))$.

According to $(3)-(7)$, one can easily check that:

$$
\begin{array}{ll}
l_{i}^{-}\left(s_{1}-s_{2}\right) \leq \hat{f}_{i}\left(s_{1}\right)-\hat{f}_{i}\left(s_{2}\right) \leq l_{i}^{+}\left(s_{1}-s_{2}\right), & \forall s_{1}, s_{2} \in \mathbb{R}, \quad(i=1, \ldots, n) \\
\sigma_{i}^{-}\left(s_{1}-s_{2}\right) \leq \hat{g}_{i}\left(s_{1}\right)-\hat{g}_{i}\left(s_{2}\right) \leq \sigma_{i}^{+}\left(s_{1}-s_{2}\right), & \forall s_{1}, s_{2} \in \mathbb{R}, \quad(i=1, \ldots, n) \\
v_{i}^{-}\left(s_{1}-s_{2}\right) \leq \hat{h}_{i}\left(s_{1}\right)-\hat{h}_{i}\left(s_{2}\right) \leq v_{i}^{+}\left(s_{1}-s_{2}\right), & \forall s_{1}, s_{2} \in \mathbb{R}, \quad(i=1, \ldots, n) \\
|\hat{Q}(t, \mathcal{E})| \leq|R \mathcal{E}| . &
\end{array}
$$

Let $\mathcal{E}(t, \phi)$, or shortly $\mathcal{E}(t)$, denote the solution of the error-state system (9) with the initial condition of the form

$$
\mathcal{E}(s)=\phi(s), s \in\left[-\tau^{*}, 0\right],
$$

where $\phi(\cdot) \in C\left(\left[-\tau^{*}, 0\right] ; \mathbb{R}^{n}\right), \tau^{*}=\max \left\{\tau_{1}, \tau_{2}\right\}$.

It is easy to see from Assumption 1 and the condition (7) that the solution of (2) exists for all $t \geq 0$ and is unique (see [9]). Furthermore, there exists a unique zero equilibrium point to the error-state system (9).

We need the following definitions to go ahead to design the desired estimators.

Definition 1: The system (8) is said to be a state estimator of the neural network (2) if the estimation error-state system (9) is asymptotically stable.

Definition 2: The system (8) is said to be an exponential state estimator of the neural network (2) if the estimation error-state system (9) is exponentially stable, i.e., there exist positive constants $k>0$ and $\mu>0$ such that every solution $\mathcal{E}(t ; \phi)$ of $(9)$ satisfies

$$
|\mathcal{E}(t)| \leq \mu \mathrm{e}^{-k t} \sup _{-\tau^{*} \leq s \leq 0}|\phi(s)|, \quad \forall t>0 .
$$

The main purpose of this paper is to establish LMI-based sufficient conditions under which the system (8) becomes a state estimator and an exponential state estimator, respectively.

\section{MAIN RESUltS AND PROOFS}

The following lemmas are essential in establishing our main results.

Lemma 1: Let $X, Y$ be any $n$-dimensional real vectors, and let $P$ be a $n \times n$ positive semi-definite matrix. Then, the following matrix inequality holds:

$$
2 X^{T} P Y \leq X^{T} P X+Y^{T} P Y .
$$

Lemma 2: (Schur Complement) Given constant matrices $\Omega_{1}, \Omega_{2}, \Omega_{3}$ where $\Omega_{1}=\Omega_{1}^{T}$ and $\Omega_{1}>0$, then

$$
\Omega_{1}+\Omega_{3}^{T} \Omega_{2}^{-1} \Omega_{3}<0
$$


if only if

$$
\left[\begin{array}{cc}
\Omega_{1} & \Omega_{3}^{T} \\
\Omega_{3} & -\Omega_{2}
\end{array}\right]<0, \quad \text { or } \quad\left[\begin{array}{cc}
-\Omega_{2} & \Omega_{3} \\
\Omega_{3}^{T} & \Omega_{1}
\end{array}\right]<0 .
$$

Lemma 3: [7] For any positive definite matrix $M>0$, scalar $\gamma>0$, vector function $\omega:[0, \gamma] \rightarrow \mathbb{R}^{n}$ such that the integrations concerned are well defined, the following inequality holds:

$$
\left(\int_{0}^{\gamma} \omega(s) d s\right)^{T} M\left(\int_{0}^{\gamma} \omega(s) d s\right) \leq \gamma\left(\int_{0}^{\gamma} \omega^{T}(s) M \omega(s) d s\right)
$$

For presentation convenience, in the following, we denote

$$
\begin{aligned}
& L_{1}=\operatorname{diag}\left(l_{1}^{+} l_{1}^{-}, \ldots, l_{n}^{+} l_{n}^{-}\right), \quad L_{2}=\operatorname{diag}\left(\frac{l_{1}^{+}+l_{1}^{-}}{2}, \ldots, \frac{l_{n}^{+}+l_{n}^{-}}{2}\right), \\
& \Sigma_{1}=\operatorname{diag}\left(\sigma_{1}^{+} \sigma_{1}^{-}, \ldots, \sigma_{n}^{+} \sigma_{n}^{-}\right), \quad \Sigma_{2}=\operatorname{diag}\left(\frac{\sigma_{1}^{+}+\sigma_{1}^{-}}{2}, \ldots, \frac{\sigma_{n}^{+}+\sigma_{n}^{-}}{2}\right), \\
& \Upsilon_{1}=\operatorname{diag}\left(v_{1}^{+} v_{1}^{-}, \ldots, v_{n}^{+} v_{n}^{-}\right), \quad \Upsilon_{2}=\operatorname{diag}\left(\frac{v_{1}^{+}+v_{1}^{-}}{2}, \ldots, \frac{v_{n}^{+}+v_{n}^{-}}{2}\right) .
\end{aligned}
$$

We are now ready to present our first main result.

Theorem 1: Under Assumption 1, the system (8) becomes a state estimator of the neural network (2) if there exist a constant $\rho>0$, a matrix $M \in \mathbb{R}^{n \times m}$, three $n \times n$ positive definite matrices $P_{1}, P_{2}, P_{3}$, and three diagonal matrices $\Lambda=\operatorname{diag}\left(\lambda_{1}, \ldots, \lambda_{n}\right)>0, \Gamma=\operatorname{diag}\left(\gamma_{1}, \ldots, \gamma_{n}\right)>0$ and $\Delta=\operatorname{diag}\left(\delta_{1}, \ldots, \delta_{n}\right)>0$ such that the following LMI holds:

$$
\Phi=\left[\begin{array}{ccccccc}
\Theta & P_{1} A+\Lambda L_{2} & \Gamma \Sigma_{2} & P_{1} B & \Delta \Upsilon_{2} & P_{1} W & M \\
A^{T} P_{1}+\Lambda L_{2} & -\Lambda & 0 & 0 & 0 & 0 & 0 \\
\Gamma \Sigma_{2} & 0 & P_{2}-\Gamma & 0 & 0 & 0 & 0 \\
B^{T} P_{1} & 0 & 0 & -P_{2} & 0 & 0 & 0 \\
\Delta \Upsilon_{2} & 0 & 0 & 0 & \tau_{2} P_{3}-\Delta & 0 & 0 \\
W^{T} P_{1} & 0 & 0 & 0 & 0 & -P_{3} & 0 \\
M^{T} & 0 & 0 & 0 & 0 & 0 & -\rho I
\end{array}\right]<0
$$

where

$$
\Theta=-P_{1} D-D^{T} P_{1}-M C-C^{T} M^{T}+\rho R^{T} R-\Lambda L_{1}-\Gamma \Sigma_{1}-\Delta \Upsilon_{1}
$$

In this case, the estimator gain matrix $K$ can be taken as

$$
K=P_{1}^{-1} M
$$

Proof: To proceed with the stability analysis of the error-state system (9), we construct the following Lyapunov-Krasovskii functional

$$
V(t)=\mathcal{E}^{T}(t) P_{1} \mathcal{E}(t)+\int_{t-\tau_{1}}^{t} \hat{G}^{T}(\mathcal{E}(s)) P_{2} \hat{G}(\mathcal{E}(s)) d s+\int_{0}^{\tau_{2}} \int_{t-s}^{t} \hat{H}^{T}(\mathcal{E}(\eta)) P_{3} \hat{H}(\mathcal{E}(\eta)) d \eta d s .
$$

The time derivative of $V(t)$ along the trajectory of the system (9) can be calculated as follows:

$$
\begin{aligned}
\dot{V}(t)= & 2 \mathcal{E}^{T}(t) P_{1}\left[(-D-K C) \mathcal{E}(t)+A \hat{F}(\mathcal{E}(t))+B \hat{G}\left(\mathcal{E}\left(t-\tau_{1}\right)\right)\right. \\
& \left.+W \int_{t-s}^{t} \hat{H}(\mathcal{E}(s)) d s-K \hat{Q}(t, \mathcal{E}(t))\right] \\
& +\hat{G}^{T}(\mathcal{E}(t)) P_{2} \hat{G}(\mathcal{E}(t))-\hat{G}^{T}\left(\mathcal{E}\left(t-\tau_{1}\right)\right) P_{2} \hat{G}\left(\mathcal{E}\left(t-\tau_{1}\right)\right) \\
& +\tau_{2} \hat{H}^{T}(\mathcal{E}(t)) P_{3} \hat{H}(\mathcal{E}(t))-\int_{t-\tau_{2}}^{t} \hat{H}^{T}(\mathcal{E}(s)) P_{3} \hat{H}(\mathcal{E}(s)) d s .
\end{aligned}
$$


It follows from (17), Lemma 1 and Lemma 2 that

$$
\begin{aligned}
-2 \mathcal{E}^{T}(t) P_{1} K \hat{Q}(t, \mathcal{E}(t)) & \leq \rho^{-1} \mathcal{E}^{T}(t) P_{1} K K^{T} P_{1}^{T} \mathcal{E}^{T}(t)+\rho \hat{Q}^{T}(t, \mathcal{E}(t)) \hat{Q}(t, \mathcal{E}(t)) \\
& \leq \rho^{-1} \mathcal{E}^{T}(t) P_{1} K K^{T} P_{1}^{T} \mathcal{E}^{T}(t)+\rho \mathcal{E}^{T}(t) R^{T} R \mathcal{E}(t) \\
-\int_{t-\tau_{2}}^{t} \hat{H}^{T}(\mathcal{E}(s)) P_{3} \hat{H}(\mathcal{E}(s)) & \leq-\left(\int_{t-\tau_{2}}^{T} \hat{H}(\mathcal{E}(s)) d s\right)^{T} P_{3} \int_{t-\tau_{2}}^{t} \hat{H}^{T}(\mathcal{E}(s)) d s .
\end{aligned}
$$

Substituting the above into (26) leads to

$$
\begin{aligned}
\dot{V}(t) \leq & 2 \mathcal{E}^{T}(t) P_{1}\left[(-D-K C) \mathcal{E}(t)+A \hat{F}(\mathcal{E}(t))+B \hat{G}\left(\mathcal{E}\left(t-\tau_{1}\right)\right)+W \int_{t-s}^{t} \hat{H}(\mathcal{E}(s)) d s\right] \\
& +\rho^{-1} \mathcal{E}^{T}(t) P_{1} K K^{T} P_{1}^{T} \mathcal{E}^{T}(t)+\rho \mathcal{E}^{T}(t) R^{T} R \mathcal{E}(t) \\
& +\hat{G}^{T}(\mathcal{E}(t)) P_{2} \hat{G}(\mathcal{E}(t))-\hat{G}^{T}\left(\mathcal{E}\left(t-\tau_{1}\right)\right) P_{2} \hat{G}\left(\mathcal{E}\left(t-\tau_{1}\right)\right) \\
& +\tau_{2} \hat{H}^{T}(\mathcal{E}(t)) P_{3} \hat{H}(\mathcal{E}(t))-\left(\int_{t-\tau_{2}}^{T} \hat{H}(\mathcal{E}(s)) d s\right)^{T} P_{3} \int_{t-\tau_{2}}^{t} \hat{H}^{T}(\mathcal{E}(s)) d s \\
\leq & X^{T}(t) \Phi_{1} X(t)+\rho^{-1} \mathcal{E}^{T}(t) P_{1} K K^{T} P_{1}^{T} \mathcal{E}(t),
\end{aligned}
$$

where

$$
\begin{aligned}
X(t):= & {\left[\begin{array}{cccccc}
\mathcal{E}^{T}(t), & \hat{F}^{T}(\mathcal{E}(t)), & \hat{G}^{T}(\mathcal{E}(t)), & \hat{G}^{T}\left(\mathcal{E}\left(t-\tau_{1}\right)\right), & \hat{H}^{T}(\mathcal{E}(t)), & \left(\int_{t-\tau_{2}}^{t} \hat{H}(\mathcal{E}(s)) d s\right)^{T}
\end{array}\right]^{T}, } \\
\Phi_{1}:= & {\left[\begin{array}{cccccc}
-P_{1} D-D^{T} P_{1}-P_{1} K C-C^{T} K^{T} P_{1}+\rho R^{T} R & P_{1} A & 0 & P_{1} B & 0 & P_{1} W \\
A^{T} P_{1} & 0 & 0 & 0 & 0 & 0 \\
0 & 0 & P_{2} & 0 & 0 & 0 \\
B^{T} P_{1} & 0 & 0 & -P_{2} & 0 & 0 \\
0 & 0 & 0 & 0 & \tau_{2} P_{3} & 0 \\
W^{T} P_{1} & 0 & 0 & 0 & 0 & -P_{3}
\end{array}\right] . }
\end{aligned}
$$

Moreover, one can infer from (14)-(15) that

$$
\begin{aligned}
& \left(\hat{f}_{i}\left(\epsilon_{i}(t)\right)-l_{i}^{+} \epsilon_{i}(t)\right)\left(\hat{f}_{i}\left(\epsilon_{i}(t)\right)-l_{i}^{-} \epsilon_{i}(t)\right) \leq 0, \quad i=1, \ldots, n, \\
& \left(\hat{g}_{i}\left(\epsilon_{i}(t)\right)-\sigma_{i}^{+} \epsilon_{i}(t)\right)\left(\hat{g}_{i}\left(\epsilon_{i}(t)\right)-\sigma_{i}^{-} \epsilon_{i}(t)\right) \leq 0, \quad i=1, \ldots, n, \\
& \left(\hat{h}_{i}\left(\epsilon_{i}(t)\right)-v_{i}^{+} \epsilon_{i}(t)\right)\left(\hat{h}_{i}\left(\epsilon_{i}(t)\right)-v_{i}^{-} \epsilon_{i}(t)\right) \leq 0, \quad i=1, \ldots, n
\end{aligned}
$$

which are equivalent to

$$
\begin{aligned}
& {\left[\begin{array}{c}
\mathcal{E}(t) \\
\hat{F}(\mathcal{E}(t))
\end{array}\right]^{T}\left[\begin{array}{cc}
l_{i}^{+} l_{i}^{-} e_{i} e_{i}^{T} & -\frac{l_{i}^{+}+l_{i}^{-}}{2} e_{i} e_{i}^{T} \\
-\frac{l_{i}^{+}+l_{i}^{-}}{2} e_{i} e_{i}^{T} & e_{i} e_{i}^{T}
\end{array}\right]\left[\begin{array}{c}
\mathcal{E}(t) \\
\hat{F}(\mathcal{E}(t))
\end{array}\right] \leq 0, \quad i=1, \ldots, n,} \\
& {\left[\begin{array}{c}
\mathcal{E}(t) \\
\hat{G}(\mathcal{E}(t))
\end{array}\right]^{T}\left[\begin{array}{cc}
\sigma_{i}^{+} \sigma_{i}^{-} e_{i} e_{i}^{T} & -\frac{\sigma_{i}^{+}+\sigma_{i}^{-}}{2} e_{i} e_{i}^{T} \\
-\frac{\sigma_{i}^{+}+\sigma_{i}^{-}}{2} e_{i} e_{i}^{T} & e_{i} e_{i}^{T}
\end{array}\right]\left[\begin{array}{c}
\mathcal{E}(t) \\
\hat{G}(\mathcal{E}(t))
\end{array}\right] \leq 0, \quad i=1, \ldots, n,} \\
& {\left[\begin{array}{c}
\mathcal{E}(t) \\
\hat{H}(\mathcal{E}(t))
\end{array}\right]^{T}\left[\begin{array}{cc}
v_{i}^{+} v_{i}^{-} e_{i} e_{i}^{T} & -\frac{v_{i}^{+}+v_{i}^{-}}{2} e_{i} e_{i}^{T} \\
-\frac{v_{i}^{+}+v_{i}^{-}}{2} e_{i} e_{i}^{T} & e_{i} e_{i}^{T}
\end{array}\right]\left[\begin{array}{c}
\mathcal{E}(t) \\
\hat{H}(\mathcal{E}(t))
\end{array}\right] \leq 0, \quad i=1, \ldots, n,}
\end{aligned}
$$

where $e_{i}$ denotes the unit column vector having " 1 " element on its $i$ th row and zeros elsewhere. 
Now, let $K=P_{1}^{-1} M$ and we have

$$
\begin{aligned}
& X^{T}(t) \Phi_{1} X(t)+\rho^{-1} \mathcal{E}^{T}(t) P_{1} K K^{T} P_{1}^{T} \mathcal{E}(t) \\
& -\sum_{i=1}^{n} \lambda_{i}\left[\begin{array}{c}
\mathcal{E}(t) \\
\hat{F}(\mathcal{E}(t))
\end{array}\right]^{T}\left[\begin{array}{cc}
l_{i}^{+} l_{i}^{-} e_{i} e_{i}^{T} & -\frac{l_{i}^{+}+l_{i}^{-}}{2} e_{i} e_{i}^{T} \\
-\frac{l_{i}^{+}+l_{i}^{-}}{2} e_{i} e_{i}^{T} & e_{i} e_{i}^{T}
\end{array}\right]\left[\begin{array}{c}
\mathcal{E}(t) \\
\hat{F}(\mathcal{E}(t))
\end{array}\right] \\
& -\sum_{i=1}^{n} \gamma_{i}\left[\begin{array}{c}
\mathcal{E}(t) \\
\hat{G}(\mathcal{E}(t))
\end{array}\right]^{T}\left[\begin{array}{cc}
\sigma_{i}^{+} \sigma_{i}^{-} e_{i} e_{i}^{T} & -\frac{\sigma_{i}^{+}+\sigma_{i}^{-}}{2} e_{i} e_{i}^{T} \\
-\frac{\sigma_{i}^{+}+\sigma_{i}^{-}}{2} e_{i} e_{i}^{T} & e_{i} e_{i}^{T}
\end{array}\right]\left[\begin{array}{c}
\mathcal{E}(t) \\
\hat{G}(\mathcal{E}(t))
\end{array}\right] \\
& -\sum_{i=1}^{n} \delta_{i}\left[\begin{array}{c}
x(t) \\
\hat{H}(\mathcal{E}(t))
\end{array}\right]^{T}\left[\begin{array}{cc}
v_{i}^{+} v_{i}^{-} e_{i} e_{i}^{T} & -\frac{v_{i}^{+}+v_{i}^{-}}{2} e_{i} e_{i}^{T} \\
-\frac{v_{i}^{+}+v_{i}^{-}}{2} e_{i} e_{i}^{T} & e_{i} e_{i}^{T}
\end{array}\right]\left[\begin{array}{c}
\mathcal{E}(t) \\
\hat{H}(\mathcal{E}(t))
\end{array}\right] \\
& =X^{T}(t) \Phi_{1} X(t)+\rho^{-1} \mathcal{E}^{T}(t) M M^{T} \mathcal{E}(t)+\left[\begin{array}{c}
\mathcal{E}(t) \\
\hat{F}(\mathcal{E}(t))
\end{array}\right]^{T}\left[\begin{array}{cc}
-\Lambda L_{1} & \Lambda L_{2} \\
\Lambda L_{2} & -\Lambda
\end{array}\right]\left[\begin{array}{c}
\mathcal{E}(t) \\
\hat{F}(\mathcal{E}(t))
\end{array}\right] \\
& +\left[\begin{array}{c}
\mathcal{E}(t) \\
\hat{G}(\mathcal{E}(t))
\end{array}\right]^{T}\left[\begin{array}{cc}
-\Gamma \Sigma_{1} & \Gamma \Sigma_{2} \\
\Gamma \Sigma_{2} & -\Gamma
\end{array}\right]\left[\begin{array}{c}
\mathcal{E}(t) \\
\hat{G}(\mathcal{E}(t))
\end{array}\right]+\left[\begin{array}{c}
\mathcal{E}(t) \\
\hat{H}(\mathcal{E}(t))
\end{array}\right]^{T}\left[\begin{array}{cc}
-\Delta \Upsilon_{1} & \Delta \Upsilon_{2} \\
\Delta \Upsilon_{2} & -\Delta
\end{array}\right]\left[\begin{array}{c}
\mathcal{E}(t) \\
\hat{H}(\mathcal{E}(t))
\end{array}\right] \\
& =X^{T}(t)\left(\Phi_{2}+\rho^{-1} \bar{M} \bar{M}^{T}\right) X(t) \text {, }
\end{aligned}
$$

where

$$
\Phi_{2}:=\left[\begin{array}{cccccc}
\Theta & P_{1} A+\Lambda L_{2} & \Gamma \Sigma_{2} & P_{1} B & \Delta \Upsilon_{2} & P_{1} W \\
A^{T} P_{1}+\Lambda L_{2} & -\Lambda & 0 & 0 & 0 & 0 \\
\Gamma \Sigma_{2} & 0 & P_{2}-\Gamma & 0 & 0 & 0 \\
B^{T} P_{1} & 0 & 0 & -P_{2} & 0 & 0 \\
\Delta \Upsilon_{2} & 0 & 0 & 0 & \tau_{2} P_{3}-\Delta & 0 \\
W^{T} P_{1} & 0 & 0 & 0 & 0 & -P_{3}
\end{array}\right], \quad \bar{M}:=\left[\begin{array}{c}
M \\
0 \\
0 \\
0 \\
0
\end{array}\right]
$$

From the condition (23) and Lemma 2 (Schur Complement), it can be concluded that

$$
\Phi_{2}+\rho \bar{M} \bar{M}^{T}<0 .
$$

Thus, from (29), (33)-(36) and (37), we obtain

$$
\begin{aligned}
\dot{V}(t) & \leq X^{T}(t) \Phi_{1} X(t)+\rho^{-1} \mathcal{E}^{T}(t) M M^{T} \mathcal{E}(t) \\
& \leq X^{T}(t)\left(\Phi_{2}+\rho^{-1} \bar{M} \bar{M}^{T}\right) X(t) \\
& \leq \lambda_{\max }\left(\Phi_{2}+\rho^{-1} \bar{M} \bar{M}^{T}\right)|X(t)|^{2} \\
& \leq \lambda_{\max }\left(\Phi_{2}+\rho^{-1} \bar{M} \bar{M}^{T}\right)|\mathcal{E}(t)|^{2} .
\end{aligned}
$$

Noticing $\lambda_{\max }\left(\Phi_{2}+\rho^{-1} \bar{M} \bar{M}^{T}\right)<0$, it follows from the Lyapunov stability theory that estimation error-state system (9) is asymptotically stable. Therefore, from Definition 1, the system (8) is a state estimator of the neural network (2).

Next, let us consider the conditions for the estimation error-state system (9) to be an exponential estimator of the neural network (2).

Theorem 2: Let $\varepsilon_{0}$ be a given positive constant and Assumption 1 hold. Then the system (8) is an exponential state estimator of the neural network (2) if there exist a constant $\rho>0$, a matrix $M \in \mathbb{R}^{n \times m}$, three $n \times n$ positive definite matrices $P_{1}, P_{2}, P_{3}$, and three diagonal matrices $\Lambda=\operatorname{diag}\left(\lambda_{1}, \ldots, \lambda_{n}\right)>0$, 
$\Gamma=\operatorname{diag}\left(\gamma_{1}, \ldots, \gamma_{n}\right)>0$ and $\Delta=\operatorname{diag}\left(\delta_{1}, \ldots, \delta_{n}\right)>0$ such that the following LMI holds:

$$
\Psi=\left[\begin{array}{ccccccc}
\Xi & P_{1} A+\Lambda L_{2} & \Gamma \Sigma_{2} & P_{1} B & \Delta \Upsilon_{2} & P_{1} W & M \\
A^{T} P_{1}+\Lambda L_{2} & -\Lambda & 0 & 0 & 0 & 0 & 0 \\
\Gamma \Sigma_{2} & 0 & \left(1+\epsilon_{0} \tau_{1}\right) P_{2}-\Gamma & 0 & 0 & 0 & 0 \\
B^{T} P_{1} & 0 & 0 & -P_{2} & 0 & 0 & 0 \\
\Delta \Upsilon_{2} & 0 & 0 & 0 & \tau_{2} P_{3}-\Delta & 0 & 0 \\
W^{T} P_{1} & 0 & 0 & 0 & 0 & -\frac{1-\epsilon_{0}}{\tau_{2}} P_{3} & 0 \\
M^{T} & 0 & 0 & 0 & 0 & 0 & -\rho I
\end{array}\right]<0
$$

where

$$
\Xi=-P_{1} D-D^{T} P_{1}-M C-C^{T} M^{T}+\rho R^{T} R-\Lambda L_{1}-\Gamma \Sigma_{1}-\Delta \Upsilon_{1}
$$

In this case, the estimator gain matrix $K$ can be determined as:

Proof: Let

$$
K=P_{1}^{-1} M
$$

$$
\begin{aligned}
\bar{V}(t)= & \mathcal{E}^{T}(t) P_{1} \mathcal{E}(t)+\int_{t-\tau_{1}}^{t} \hat{G}^{T}(\mathcal{E}(s)) P_{2} \hat{G}(\mathcal{E}(s)) d s,+\epsilon_{0} \int_{0}^{\tau_{1}} \int_{t-s}^{t} \hat{G}^{T}(\mathcal{E}(\eta)) P_{2} \hat{G}(\mathcal{E}(\eta)) d \eta d s \\
& +\int_{0}^{\tau_{2}} \int_{t-s}^{t} \hat{H}^{T}(\mathcal{E}(\eta)) P_{3} \hat{H}(\mathcal{E}(\eta)) d \eta d s .
\end{aligned}
$$

Similar to the derivation of Theorem 1, the time derivative of $\bar{V}$ along the system (9) can be calculated as follows:

$$
\begin{aligned}
\frac{d}{d t} \bar{V}(t) \leq & 2 \mathcal{E}^{T}(t) P_{1}\left((-D-K C) \mathcal{E}(t)+A \hat{F}(\mathcal{E}(t))+B \hat{G}\left(\mathcal{E}\left(t-\tau_{1}\right)\right)+W \int_{t-\tau_{2}}^{t} \hat{H}(\mathcal{E}(s)) d s\right) \\
& +\rho^{-1} \mathcal{E}^{T}(t) P_{1} K K^{T} P_{1}^{T} \mathcal{E}^{T}(t)+\rho \mathcal{E}^{T}(t) R^{T} R \mathcal{E}(t) \\
& +\left(1+\epsilon_{0} \tau_{1}\right) \hat{G}^{T}(\mathcal{E}(t)) P_{2} \hat{G}(\mathcal{E}(t))-\hat{G}^{T}\left(\mathcal{E}\left(t-\tau_{1}\right)\right) P_{2} \hat{G}\left(\mathcal{E}\left(t-\tau_{1}\right)\right) \\
& +\tau_{2} \hat{H}^{T}(\mathcal{E}(t)) P_{3} \hat{H}(\mathcal{E}(t))-\frac{1-\epsilon_{0}}{\tau_{2}}\left(\int_{t-\tau_{2}}^{t} \hat{H}(\mathcal{E}(s)) d s\right)^{T} P_{3}\left(\int_{t-\tau_{2}}^{t} \hat{H}(\mathcal{E}(s)) d s\right) \\
& -\epsilon_{0} \int_{t-\tau_{1}}^{t} \hat{G}^{T}(\mathcal{E}(s)) P_{2} \hat{G}(\mathcal{E}(s)) d s-\epsilon_{0} \int_{t-\tau_{2}}^{t} \hat{H}^{T}(\mathcal{E}(s)) P_{3} \hat{H}(\mathcal{E}(s)) d s \\
= & Y^{T}(t) \Psi_{1} Y(t)+\rho^{-1} \mathcal{E}^{T}(t) P_{1} K K^{T} P_{1}^{T} \mathcal{E}^{T}(t) \\
& -\epsilon_{0} \int_{t-\tau_{1}}^{t} \hat{G}^{T}(\mathcal{E}(s)) P_{2} \hat{G}(\mathcal{E}(s)) d s-\epsilon_{0} \int_{t-\tau_{2}}^{t} \hat{H}^{T}(\mathcal{E}(s)) P_{3} \hat{H}(\mathcal{E}(s)) d s,
\end{aligned}
$$

where

$$
\begin{aligned}
Y(t) & =\left[\begin{array}{lcccccc}
\mathcal{E}^{T}(t) & \hat{F}^{T}(\mathcal{E}(t)) & \hat{G}^{T}(\mathcal{E}(t)) & \hat{G}^{T}\left(\mathcal{E}\left(t-\tau_{1}\right)\right) & \hat{H}^{T}(\mathcal{E}(t)) & \int_{t-\tau_{2}}^{t} \hat{H}(\mathcal{E}(s)) d s
\end{array}\right]^{T}, \\
\Psi_{1} & =\left[\begin{array}{cccccc}
-P_{1} D-D^{T} P_{1}-P_{1} K C-C^{T} K^{T} P_{1}+\rho R^{T} R & P_{1} A & 0 & P_{1} B & 0 & P_{1} W \\
A^{T} P_{1} & 0 & 0 & 0 & 0 & 0 \\
0 & 0 & \left(1+\epsilon_{0} \tau_{1}\right) P_{2} & 0 & 0 & 0 \\
B^{T} P_{1} & 0 & 0 & -P_{2} & 0 & 0 \\
0 & 0 & 0 & 0 & \tau_{2} P_{3} & 0 \\
W^{T} P_{1} & 0 & 0 & 0 & 0 & -\frac{1-\epsilon_{0}}{\tau_{2}} P_{3}
\end{array}\right] .
\end{aligned}
$$


Furthermore, by (14)-(16) and $K=P_{1}^{-1} M$, we have

$$
\begin{aligned}
& Y^{T}(t) \Psi_{1} Y(t)+\rho^{-1} \mathcal{E}^{T}(t) P_{1} K K^{T} P_{1}^{T} \mathcal{E}(t) \\
& -\sum_{i=1}^{n} \lambda_{i}\left[\begin{array}{c}
\mathcal{E}(t) \\
\hat{F}(\mathcal{E}(t))
\end{array}\right]^{T}\left[\begin{array}{cc}
l_{i}^{+} l_{i}^{-} e_{i} e_{i}^{T} & -\frac{l_{i}^{+}+l_{i}^{-}}{2} e_{i} e_{i}^{T} \\
-\frac{l_{i}^{+}+l_{i}^{-}}{2} e_{i} e_{i}^{T} & e_{i} e_{i}^{T}
\end{array}\right]\left[\begin{array}{c}
\mathcal{E}(t) \\
\hat{F}(\mathcal{E}(t))
\end{array}\right] \\
& -\sum_{i=1}^{n} \gamma_{i}\left[\begin{array}{c}
\mathcal{E}(t) \\
\hat{G}(\mathcal{E}(t))
\end{array}\right]^{T}\left[\begin{array}{cc}
\sigma_{i}^{+} \sigma_{i}^{-} e_{i} e_{i}^{T} & -\frac{\sigma_{i}^{+}+\sigma_{i}^{-}}{2} e_{i} e_{i}^{T} \\
-\frac{\sigma_{i}^{+}+\sigma_{i}^{-}}{2} e_{i} e_{i}^{T} & e_{i} e_{i}^{T}
\end{array}\right]\left[\begin{array}{c}
\mathcal{E}(t) \\
\hat{G}(\mathcal{E}(t))
\end{array}\right] \\
& -\sum_{i=1}^{n} \delta_{i}\left[\begin{array}{c}
\mathcal{E}(t) \\
\hat{H}(\mathcal{E}(t))
\end{array}\right]^{T}\left[\begin{array}{cc}
v_{i}^{+} v_{i}^{-} e_{i} e_{i}^{T} & -\frac{v_{i}^{+}+v_{i}^{-}}{2} e_{i} e_{i}^{T} \\
-\frac{v_{i}^{+}+v_{i}^{-}}{2} e_{i} e_{i}^{T} & e_{i} e_{i}^{T}
\end{array}\right]\left[\begin{array}{c}
\mathcal{E}(t) \\
\hat{H}(\mathcal{E}(t))
\end{array}\right] \\
& =\eta^{T}(t) \Psi_{1} \eta(t)+\rho^{-1} \mathcal{E}^{T}(t) M M^{T} \mathcal{E}(t)+\left[\begin{array}{c}
\mathcal{E}(t) \\
\hat{F}(\mathcal{E}(t))
\end{array}\right]^{T}\left[\begin{array}{cc}
-\Lambda L_{1} & \Lambda L_{2} \\
\Lambda L_{2} & -\Lambda
\end{array}\right]\left[\begin{array}{c}
\mathcal{E}(t) \\
\hat{F}(\mathcal{E}(t))
\end{array}\right] \\
& +\left[\begin{array}{c}
\mathcal{E}(t) \\
\hat{G}(\mathcal{E}(t))
\end{array}\right]^{T}\left[\begin{array}{cc}
-\Gamma \Sigma_{1} & \Gamma \Sigma_{2} \\
\Gamma \Sigma_{2} & -\Gamma
\end{array}\right]\left[\begin{array}{c}
\mathcal{E}(t) \\
\hat{G}(\mathcal{E}(t))
\end{array}\right]+\left[\begin{array}{c}
\mathcal{E}(t) \\
\hat{H}(\mathcal{E}(t))
\end{array}\right]^{T}\left[\begin{array}{cc}
-\Delta \Upsilon_{1} & \Delta \Upsilon_{2} \\
\Delta \Upsilon_{2} & -\Delta
\end{array}\right]\left[\begin{array}{c}
\mathcal{E}(t) \\
\hat{H}(\mathcal{E}(t))
\end{array}\right] \\
& =Y^{T}(t)\left[\Psi_{2}+\rho^{-1} \bar{M} \bar{M}^{T}\right] Y(t)
\end{aligned}
$$

where

$$
\Psi_{2}=\left[\begin{array}{cccccc}
\Pi & P_{1} A+\Lambda L_{2} & \Gamma \Sigma_{2} & P_{1} B & \Delta \Upsilon_{2} & P_{1} W \\
A^{T} P_{1}+\Lambda L_{2} & -\Lambda & 0 & 0 & 0 & 0 \\
\Gamma \Sigma_{2} & 0 & \left(1+\epsilon_{0} \tau_{1}\right) P_{2}-\Gamma & 0 & 0 & 0 \\
B^{T} P_{1} & 0 & 0 & -P_{2} & 0 & 0 \\
\Delta \Upsilon_{2} & 0 & 0 & 0 & \tau_{2} P_{3}-\Delta & 0 \\
W^{T} P_{1} & 0 & 0 & 0 & 0 & -\frac{1-\epsilon_{0}}{\tau_{2}} P_{3}
\end{array}\right], \quad \bar{M}=\left[\begin{array}{c}
M \\
0 \\
0 \\
0 \\
0
\end{array}\right] .
$$

Again, by Lemma 2, the condition (39) is equivalent to

$$
\Psi_{2}+\rho^{-1} \bar{M} \bar{M}^{T}<0,
$$

which implies from (45) that

$$
\begin{aligned}
& Y^{T}(t) \Psi_{1} Y(t)+\rho^{-1} \mathcal{E}^{T}(t) M M^{T} \mathcal{E}(t) \\
\leq & \lambda_{\max }\left(\Psi_{2}+\rho^{-1} \bar{M} \bar{M}^{T}\right)|Y(t)|^{2} \\
\leq & \lambda_{\max }\left(\Psi_{2}+\rho^{-1} \bar{M} \bar{M}^{T}\right)|\mathcal{E}(t)|^{2}
\end{aligned}
$$

Hence, it follows from (42) and (47) that

$$
\begin{aligned}
\frac{d}{d t} \bar{V}(t) \leq & Y^{T}(t) \Psi_{1} Y(t)+\rho^{-1} \mathcal{E}^{T}(t) M M^{T} \mathcal{E}^{T}(t) \\
& \quad-\epsilon_{0} \int_{t-\tau_{1}}^{t} \hat{G}^{T}(\mathcal{E}(s)) P_{2} \hat{G}(\mathcal{E}(s)) d s-\epsilon_{0} \int_{t-\tau_{2}}^{t} \hat{H}^{T}(\mathcal{E}(s)) P_{3} \hat{H}(\mathcal{E}(s)) d s \\
\leq & \lambda_{\max }\left(\Psi_{2}+\rho^{-1} \bar{M} \bar{M}^{T}\right)|\mathcal{E}(t)|^{2}-\epsilon_{0} \int_{t-\tau_{1}}^{t} \hat{G}^{T}(\mathcal{E}(s)) P_{2} \hat{G}(\mathcal{E}(s)) d s \\
& -\epsilon_{0} \int_{t-\tau_{2}}^{t} \hat{H}^{T}(\mathcal{E}(s)) P_{3} \hat{H}(\mathcal{E}(s)) d s .
\end{aligned}
$$

Also, along the line of the proof of Theorem 1 in [13], one can infer that

$$
\bar{V}(t) \leq \lambda_{\max }\left(P_{1}\right)|\mathcal{E}(t)|^{2}+\left(1+\epsilon_{0} \tau_{1}\right) \int_{t-\tau_{1}}^{t} \hat{G}^{T}(\mathcal{E}(s)) P_{2} \hat{G}(\mathcal{E}(s)) d s+\tau_{2} \int_{t-\tau_{2}}^{t} \hat{H}^{T}(\mathcal{E}(s)) P_{3} \hat{H}(\mathcal{E}(s)) d s
$$


In order to analyze the exponential stability of the state-error system (9), we consider the following modified Lyapunov-Krasovskii functional:

$$
\hat{V}(t)=\mathrm{e}^{2 k t} \bar{V}(t)
$$

where $k$ is a positive constant to be determined.

Calculating the time derivative of $\hat{V}(t)$ along trajectory of the system (9) and using (48) and (49), we obtain:

$$
\begin{aligned}
\frac{d}{d t} \hat{V}(t)= & 2 k \mathrm{e}^{2 k t} \bar{V}(t)+\mathrm{e}^{2 k t} \frac{d}{d t} \bar{V}(t) \\
\leq & 2 k \mathrm{e}^{2 k t}\left[\lambda_{\max }\left(P_{1}\right)|\mathcal{E}(t)|^{2}+\left(1+\epsilon_{0} \tau_{1}\right) \int_{t-\tau_{1}}^{t} \hat{G}^{T}(\mathcal{E}(s)) P_{2} \hat{G}(\mathcal{E}(s)) d s\right. \\
& \left.+\tau_{2} \int_{t-\tau_{2}}^{t} \hat{H}^{T}(\mathcal{E}(s)) P_{3} \hat{H}(\mathcal{E}(s)) d s\right]+\mathrm{e}^{2 k t}\left[\lambda_{\max }\left(\Psi_{2}+\rho^{-1} \bar{M} \bar{M}^{T}\right)|\mathcal{E}(t)|^{2}\right. \\
& \left.-\epsilon_{0} \int_{t-\tau_{1}}^{t} \hat{G}^{T}(\mathcal{E}(s)) P_{2} \hat{G}(\mathcal{E}(s)) d s-\epsilon_{0} \int_{t-\tau_{2}}^{t} \hat{H}^{T}(\mathcal{E}(s)) P_{3} \hat{H}(\mathcal{E}(s)) d s\right] \\
\leq & \mathrm{e}^{2 k t}\left[\left(2 k \lambda_{\max }\left(P_{1}\right)+\lambda_{\max }\left(\Psi_{2}+\rho^{-1} \bar{M} \bar{M}^{T}\right)\right)|\mathcal{E}(t)|^{2}+\left(2 k\left(1+\epsilon_{0} \tau_{1}\right)-\epsilon_{0}\right) \int_{t-\tau_{1}}^{t} \hat{G}^{T}(x(s)) P_{2} \hat{G}(\mathcal{E}(s)) d s\right. \\
& \left.+\left(2 k \tau_{2}-\epsilon_{0}\right) \int_{t-\tau_{2}}^{t} \hat{H}^{T}(\mathcal{E}(s)) P_{3} \hat{H}(\mathcal{E}(s)) d s\right]
\end{aligned}
$$

Set

$$
k_{0}=\min \left\{-\frac{\lambda_{\max }\left(\Psi_{2}+\rho^{-1} \bar{M} \bar{M}^{T}\right)}{2 \lambda_{\max }\left(P_{1}\right)}, \frac{\epsilon_{0}}{2\left(1+\epsilon_{0} \tau_{1}\right)}, \frac{\epsilon_{0}}{2 \tau_{2}}\right\},
$$

and fix $k$ to be a positive constant satisfying

$$
k \leq k_{0}
$$

We can now obtain from (51) that

$$
\frac{d}{d t} \hat{V}(t) \leq 0
$$

which, together with (41) and (49), implies that

$$
\begin{aligned}
\hat{V}(t) \leq & \hat{V}(0)=\bar{V}(0) \\
\leq & \lambda_{\max }\left(P_{1}\right)|\mathcal{E}(0)|^{2}+\left(1+\epsilon_{0} \tau_{1}\right) \lambda_{\max }\left(P_{2}\right) \int_{-\tau_{1}}^{0}|\hat{G}(\mathcal{E}(s))|^{2} d s \\
& \quad+\tau_{2} \lambda_{\max }\left(P_{3}\right) \int_{-\tau_{2}}^{0}|\hat{H}(\mathcal{E}(s))|^{2} d s .
\end{aligned}
$$

Let

$$
\begin{aligned}
& \sigma=\max _{1 \leq i \leq n}\left\{\left|\sigma_{i}^{-}\right|,\left|\sigma_{i}^{+}\right|\right\}, v=\max _{1 \leq i \leq n}\left\{\left|v_{i}^{-}\right|,\left|v_{i}^{+}\right|\right\} \\
& \mu_{0}=\lambda_{\max }\left(P_{1}\right)+\left(1+\epsilon_{0} \tau_{1}\right) \tau_{1} \sigma^{2} \lambda_{\max }\left(P_{2}\right)+\tau_{2}^{2} v^{2} \lambda_{\max }\left(P_{3}\right) .
\end{aligned}
$$

Then, it is indicated from (54) that

$$
\begin{aligned}
\mathrm{e}^{2 k t} \bar{V}(t) & \leq \lambda_{\max }\left(P_{1}\right)|\mathcal{E}(0)|^{2}+\left(1+\epsilon_{0} \tau_{1}\right) \tau_{1} \sigma^{2} \lambda_{\max }\left(P_{2}\right) \sup _{-\tau_{1} \leq s \leq 0}|\mathcal{E}(s)|^{2}+\tau_{2}^{2} v^{2} \lambda_{\max }\left(P_{3}\right) \sup _{-\tau_{2} \leq s \leq 0}|\mathcal{E}(s)|^{2} \\
& \leq\left(\lambda_{\max }\left(P_{1}\right)+\left(1+\epsilon_{0} \tau_{1}\right) \tau_{1} \sigma^{2} \lambda_{\max }\left(P_{2}\right)+\tau_{2}^{2} v^{2} \lambda_{\max }\left(P_{3}\right)\right) \sup _{-\tau^{*} \leq s \leq 0}|\mathcal{E}(s)|^{2} \\
& =\mu_{0} \sup _{-\tau^{*} \leq s \leq 0}|\mathcal{E}(s)|^{2}=\mu_{0} \sup _{-\tau^{*} \leq s \leq 0}|\phi(s)|^{2}
\end{aligned}
$$


and therefore

$$
\bar{V}(t) \leq \mu_{0} \mathrm{e}^{-2 k t}|\phi(s)|^{2}
$$

Noticing $\hat{V}(t) \geq \lambda_{\max }\left(P_{1}\right)|\mathcal{E}(t)|^{2}$, we obtain

$$
|\mathcal{E}(t)|^{2} \leq \frac{\mu_{0}}{\lambda_{\max }\left(P_{1}\right)} \mathrm{e}^{-2 k t} \sup _{-\tau^{*} \leq s \leq 0}|\phi(s)|^{2}
$$

and hence

$$
|x(t)| \leq \mu \mathrm{e}^{-k t} \sup _{-\tau^{*} \leq s \leq 0}\left|\phi(s)-u^{*}\right|,
$$

where $\mu=\sqrt{\frac{\mu_{0}}{\lambda_{\max \left(P_{1}\right)}}}$. From Definition 2, the proof of this theorem is complete.

Remark 2: In Theorem 1 and Theorem 2, sufficient conditions are provided for the system (2) to be globally asymptotically and exponentially stable, respectively. Such conditions are expressed in the form of LMIs, which could be easily checked by utilizing the recently developed interior-point methods available in Matlab toolbox, and no turning of parameters will be needed [6]. It should be mentioned that, in the past decade, LMIs have gained much attention for their computational tractability and usefulness in many areas because the so-called interior point method (see [6]) has been proven to be numerically very efficient for solving the LMIs.

\section{Numerical EXAMPle}

In this section, we present a simulation example so as to illustrate the usefulness of our main results. Consider a 3-neuron neural network (2) with the following parameters:

$$
\begin{aligned}
& D=\left[\begin{array}{ccc}
4.5 & 0 & 0 \\
0 & 5 & 0 \\
0 & 0 & 6
\end{array}\right], A=\left[\begin{array}{ccc}
1 & -0.6 & 0.8 \\
0.4 & -1.5 & 0.6 \\
-0.7 & -1.1 & -1.2
\end{array}\right], B=\left[\begin{array}{ccc}
-1.2 & 0.8 & 0.6 \\
-0.5 & 1.1 & 0.7 \\
0.6 & -0.8 & 1.2
\end{array}\right], \\
& W=\left[\begin{array}{ccc}
1.5 & 0.6 & -0.9 \\
0.7 & 1.2 & 1.2 \\
-0.5 & -0.6 & 1.3
\end{array}\right], I(t)=\left[\begin{array}{c}
5+5 \sin t \\
5 \cos t \\
5 \sin t
\end{array}\right], \tau_{1}=0.1, \quad \tau_{2}=0.2 .
\end{aligned}
$$

Take the activation function as follows:

$$
\begin{aligned}
& f_{1}(s)=g_{1}(s)=h_{1}(s)=\tanh (-1.2 s) \\
& f_{2}(s)=g_{2}(s)=h_{2}(s)=\tanh (1.4 s), \\
& f_{3}(s)=g_{3}(s)=h_{3}(s)=\tanh (-2.4 s), s \in \mathbb{R} .
\end{aligned}
$$

and assume that, for the network output (6), the parameters are given as:

$$
C=\left[\begin{array}{lll}
1 & 0 & 1 \\
0 & 1 & 1
\end{array}\right], \quad Q(x)=\left[\begin{array}{l}
0.2 \sin x_{1} \\
0.2 \cos x_{2}
\end{array}\right] .
$$

It can be readily verified that

$$
L_{1}=\Sigma_{1}=\Upsilon_{1}=\left[\begin{array}{lll}
0 & 0 & 0 \\
0 & 0 & 0 \\
0 & 0 & 0
\end{array}\right], \quad L_{2}=\Sigma_{2}=\Upsilon_{2}=\left[\begin{array}{ccc}
-0.6 & 0 & 0 \\
0 & 0.7 & 0 \\
0 & 0 & -1.2
\end{array}\right]
$$

and

$$
R=\left[\begin{array}{ccc}
0.2 & 0 & 0 \\
0 & 0.2 & 0 \\
0 & 0 & 0
\end{array}\right]
$$


Now, let $\epsilon_{0}=0.01$. Using the Matlab LMI toolbox to solve the LMI (39), we obtain

$$
\begin{aligned}
& P_{1}=\left[\begin{array}{ccc}
0.2188 & -0.0379 & -0.0243 \\
-0.0379 & 0.3194 & -0.1065 \\
-0.0243 & -0.1065 & 0.2980
\end{array}\right], \quad P_{2}=\left[\begin{array}{ccc}
0.5258 & -0.0475 & 0.0670 \\
-0.0475 & 0.6785 & -0.0541 \\
0.0670 & -0.0541 & 0.1560
\end{array}\right] \text {, } \\
& P_{3}=\left[\begin{array}{ccc}
0.3994 & 0.1139 & -0.0336 \\
0.1139 & 0.4032 & -0.0496 \\
-0.0336 & -0.0496 & 0.2633
\end{array}\right], \quad \Lambda=\left[\begin{array}{ccc}
0.9154 & 0 & 0 \\
0 & 0.6932 & 0 \\
0 & 0 & 0.5755
\end{array}\right] \text {, } \\
& \Gamma=\left[\begin{array}{ccc}
1.1356 & 0 & 0 \\
0 & 1.3852 & 0 \\
0 & 0 & 0.3540
\end{array}\right], \quad \Delta=\left[\begin{array}{ccc}
0.4030 & 0 & 0 \\
0 & 0.4538 & 0 \\
0 & 0 & 0.1502
\end{array}\right] \text {, } \\
& M=\left[\begin{array}{ll}
0.0854 & 0.0903 \\
0.2633 & 0.0668 \\
0.2331 & 0.4325
\end{array}\right], \quad \rho=0.9125, \quad K=P_{1}^{-1} M=\left[\begin{array}{ll}
0.7736 & 0.7759 \\
1.3602 & 0.9156 \\
1.3314 & 1.8419
\end{array}\right] \text {. }
\end{aligned}
$$

Therefore, it follows from Theorem 2 that the system (8) is an estimator of the neural network (2). Such a conclusion is further supported by the simulation results given in Figs. 1-3.

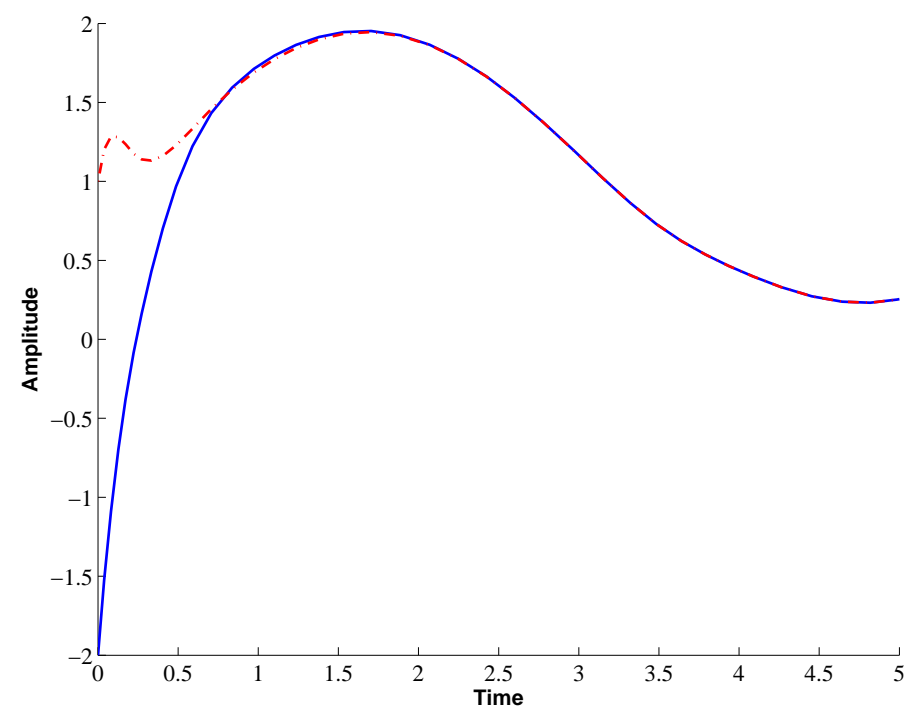

Fig. 1. The True state $x_{1}$ (solid) and its Estimate (dashed)

\section{Conclusions}

In this paper, we have studied the state estimation problem for a class of recurrent neural networks with mixed discrete and distributed delays, where we don't need the activation functions to be monotonic, or differentiable, or bounded. An exponential state estimator is designed to estimate the neuron states, through available output measurements, such that the dynamics of the estimation error is globally exponentially stable. By using the Laypunov-Krasovskii functional, we have established an LMI approach to derive the sufficient conditions guaranteeing the existence of the state estimators. The explicit expression of the desired estimator has been parameterized by means of the solution to an LMI. A simulation example has been used to illustrate the usefulness of the derived LMI-based stability conditions. One of the future research topics would be the extension of the present results to more general cases, for example, the case that there exist parameter uncertainties, the case that the neural network is inherently stochastic, and the case where the network modes are subjected to Markovian switching. The results will appear in the near future. 


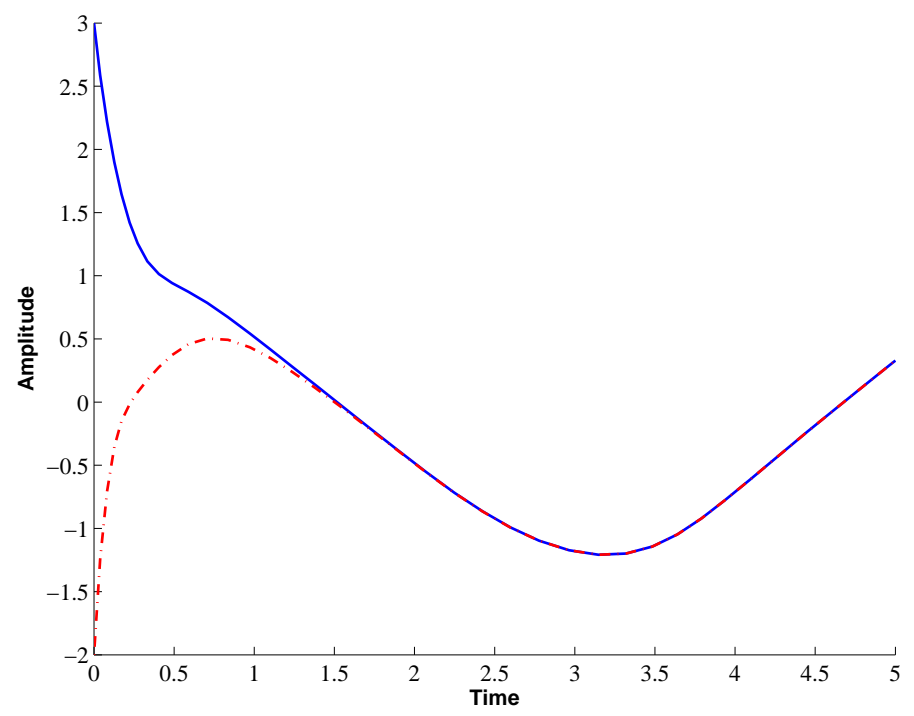

Fig. 2. The True state $x_{2}$ (solid) and its estimate (dashed)

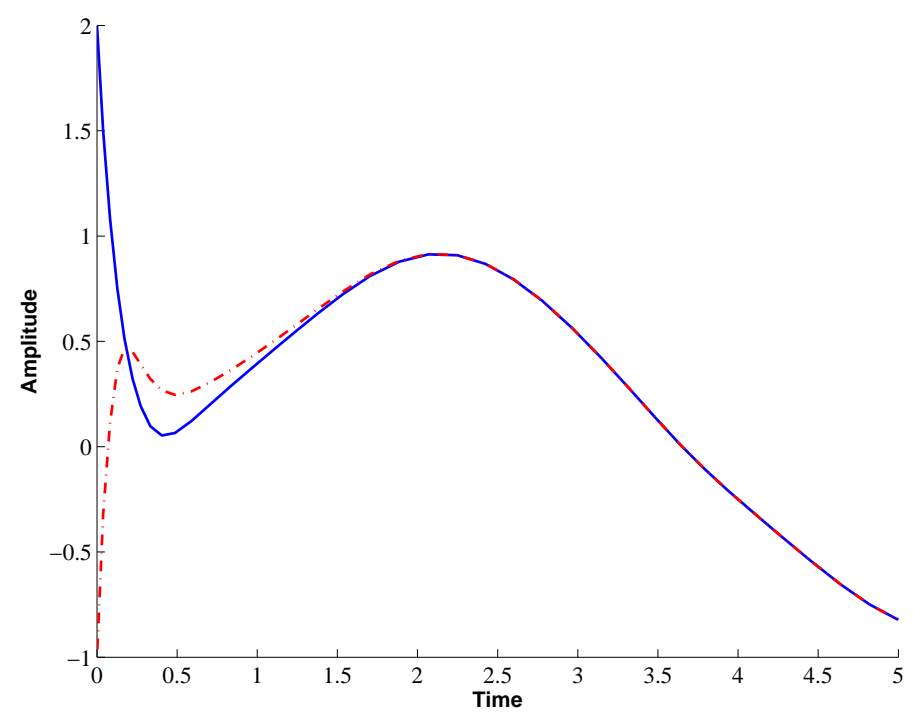

Fig. 3. The True state $x_{3}$ (solid) and its estimate (dashed)

\section{REFERENCES}

[1] Arik, S. (2000) Stability analysis of delayed neural networks. IEEE Transactions on Circuits Systems -I, 47, 1089-1092.

[2] Cao, J., Li, H.X. and Han, L. (2006) Novel results concerning global robust stability of delayed neural networks. Nonlinear Analysis: Real World Applications, 7(3), 458-469.

[3] Cao, J., Huang, D.-S. and Qu, Y. (2005) Global robust stability of delayed recurrent neural networks. Chaos, Solitons E6 Fractals, 23(1), 221-229.

[4] Cao, J. and Ho, D. W. C. (2005) A general framework for global asymptotic stability analysis of delayed neural networks based on LMI approach. Chaos, Solitons $\&$ Fractals, 24(5), 1317-1329.

[5] Elanayar, V. T. S. and Shin, Y. C. (1994) Approximation and estimation of nonlinear stochastic dynamic systems using radial basis function neural networks. IEEE Trans. Neural Networks, 5(4), 594-603.

[6] Gahinet, P., Nemirovsky, A., Laub, A. J. and Chilali, M. (1995) LMI control toolbox: for use with Matlab, The MATH Works Inc.

[7] Gu, K. (2000) An integral inequality in the stability problem of time-delay systems. In: Proceedings of 39th IEEE Conference on Decision and Control, December 2000, Sydney, Australia, 2805-2810.

[8] Habtom, R. and Litz, L. (1997) Estimation of unmeasured inputs using recurrent neural networks and the extended Kalman filter. In: Proc. International Conference on Neural Networks. 4, 2067-2071, Houston, U.S.A. 
[9] Hale J. K. (1977) The theory of functional differential equations. New York: Springer-Verlag.

[10] Huang, H. and Cao, J. (2003) On global asymptotic stability of recurrent neural networks with time-varying delays. Applied Mathematics and Computation, 142(1), 143-154.

[11] Huang, H., Qu, Y. and Li, H.X. (2005) Robust stability analysis of switched Hopfield neural networks with time-varying delay under uncertainty. Physics Letters A, 345(4-6), 345-354.

[12] Joy, M. P. (2000) Results concerning the absolute stability of delayed neural networks. Neural Networks, $13,613-616$.

[13] Liu, Y., Wang, Z. and Liu, X. (2006) Global exponential stability of generalized recurrent nueral networks with discrete and distributed delays. Neural Networks, 19(5), 667-675.

[14] Salam, F. M. and Zhang, J. (2001) Adaptive neural observer with forward co-state propagation. In: Proc. International Joint Conference on Neural Networks (IJCNN '01), 1, 675-680, Washington, U.S.A.

[15] Singh, V. (2006) Simplified LMI condition for global asymptotic stability of delayed neural networks. Chaos, Solitons E6 Fractals, 29(2), 470-473.

[16] Song, Q., Zhao, Z. and Li, Y. (2005) Global exponential stability of BAM neural networks with distributed delays and reaction-diffusion terms. Physics Letters A, 335(2-3), 213-225.

[17] Song, Q. and Zhao, Z. (2005) Global dissipativity of neural networks with both variable and unbounded delays. Chaos, Solitons $\& 5$ Fractals, 25(2), 393-401.

[18] Wang, Z., Ho, D. W. C. and Liu, X. (2005) State estimation for delayed neural networks. IEEE Trans. Neural Networks, 16(1), 279-284.

[19] Wang, Z., Liu, Y. and Liu, X. (2005) On global asymptotic stability of neural networks with discrete and distributed delays. Physics Letters A, 345(4-6), 299-308.

[20] Wang, Z., Liu, Y., Fraser, K. and Liu, X. (2006) Stochastic stability of uncertain Hopfield neural networks with discrete and distributed delays. Physics Letters A, 354(4), 288-297.

[21] Wang, Z., Liu, Y., Li, M. and Liu, X. (2006) Stability analysis for stochastic Cohen-Grossberg neural networks with mixed time delays. IEEE Trans. Neural Networks, 17(3), 814-820.

[22] Wang, Z., Shu, H., Liu, Y., Ho, D.W.C. and Liu, X. (2006) Robust stability analysis of generalized neural networks with discrete and distributed time delays. Chaos, Solitons and Fractals, 30(4), 886-896.

[23] Zhao, H. (2004) Global asymptotic stability of Hopfield neural network involving distributed delays. Neural Networks, 17, 47-53.

[24] Zhao, H. (2005) Global exponential stability and periodicity of cellular neural networks with variable delays. Physics Letters A, 336, 331-341. 